\title{
Categorías bioéticas en la interpretación de ser mujer con morbilidad materna extrema ${ }^{\dagger}$
}

\author{
Bioethic categories for the interpretation of being a woman with extreme maternal \\ morbidity
}

\section{Categorias bioéticas na interpretação do ser mulher com extrema morbidade materna}

\author{
Mónica Paola Quemba-Mesal
}

\section{Resumen}

En el presente artículo de reflexión derivado de una investigación, se discute en torno a las categorías de análisis bioético que facilitan la interpretación de la experiencia vivida al ser mujer en condición de morbilidad materna extrema, siendo quien tiene que enfrentarse a esta situación. Este estudio se basa para su realización en los siguientes indicadores: la equidad, la autonomía, la justicia, la concepción del bien y el mal, el acceso a los servicios de salud, las condiciones de vida desde la pluralidad cultural, la comunicación, el lenguaje y la deliberación bioética; constituyéndose esta última en una herramienta que permite concretar estas categorías a problemas de la vida cotidiana. Estas categorías de análisis desempeñan un rol central, al facilitar una interpretación de diferentes problemáticas en torno a la vida, teniendo como cimiento dialógico y orientador a la bioética.

Palabras clave: Bioética, salud materna, justicia social, equidad en salud.

\begin{abstract}
In this reflection paper, derived from research, the categories of bioethical analysis that contribute to the interpretation of the experience of being a woman with extreme maternal morbidity are discussed. Categories with highest potential toward this interpretation are equity, autonomy, justice, conception of good and evil, access to healthcare services, life conditions from a cultural pluralism perspective, communication and language, and bioethical dilemma, the last one being a tool that allows to focus these categories on concrete problems. These categories of analysis have a key role, allowing the interpretation of different life issues using bioethics as a dialogic and orientating foundation.
\end{abstract}

Keywords: Bioethics, maternal health, social justice, health equity.

\section{Resumo}

O presente artigo de reflexão produto de pesquisa, discute ao redor das categorias de analise bioética que contribui na interpretação da experiência vivida ao ser mulher na condição de morbidade materna extrema, sendo as que têm maior potencialidade perante a interpretação de equidade, autonomia, justiça e conceito do bem e do mal, acesso aos serviços de saúde, condições de vida desde a diversidade cultural, a comunicação e a linguagem, a deliberação bioética, sendo esta última uma ferramenta que permite relacionar essas categorias com problemáticas concretas. Essas categorias de analise desempenham um papel central, permitindo

\section{Autor de correspondencia}

${ }^{1}$ Enfermera, Especialista en Epidemiología Clínica. Magíster en Enfermería y Estudiante Doctorado en Bioética. Docente Universidad de Boyacá. Tunja, Boyacá - Colombia. Correo: mpquemba@uniboyaca.edu.co (1) 0000-00015646-6123

†Este manuscrito de reflexión derivado de una investigación, hace parte de los productos de la Tesis de Doctorado en Bioética en la Universidad Militar Nueva Granada, titulada: "Significados de la experiencia vivida al ser mujer en condición de Morbilidad Materna Extrema (MME). Un análisis desde la bioética de las poblaciones y de intervención por medio de la fenomenología médica"

Recibido: 21 octubre 2020

Aprobado: 2 marzo 2021

Para citar este artículo / To reference this article / Para citar este artigo:

Quemba-Mesa MP. Categorías bioéticas en la interpretación de ser mujer con morbilidad materna extrema. Rev. cienc. cuidad. 2021; 18(2):120-129. https:// doi.org/10.22463/17949831.2742

(C) Universidad Francisco de Paula Santander. Este es un artículo bajo la licencia CC-BY-NC-ND 
Scientific Journal of Nursing

a interpretação a diferentes problemas relacionados com a vida, tendo como base dialógica e orientadora a bioética.

Palavras-chave: Bioética, Saúde materna, Justiça Social, Equidade em saúde.

\section{Introducción}

Este artículo se estructura en torno al desarrollo del interés investigativo que ocupa la tesis Doctoral en Bioética en la Universidad Militar Nueva Granada, centrada en interpretar a la luz de la bioética los significados de la experiencia vivida al ser mujer en condición de Morbilidad Materna Extrema (MME). Al respecto, se tienen en cuenta conceptos imprescindibles para el desarrollo de esta interpretación sobre la corporalidad, la memoria y los significados que las mujeres sobrevivientes atribuyen a sus experiencias durante la pre-concepción, la gestación, el nacimiento de su hijo y la atención en salud, durante la vivencia de estos eventos agudos que la hicieron estar cerca de la muerte.

Al hablar de bioética, es preciso mencionar que esta tiene diferentes alcances, que no solamente se ocupan del campo netamente clínico y médico, sino que, desde un enfoque global, se preocupa por todas las problemáticas en torno a la vida, incluyendo la vida no humana y el medio ambiente. Al respecto, Osorio-García SN explica que hoy la bioética hace una búsqueda razonable de una nueva sociedad que posibilite para todos los habitantes de la tierra una vida decente y digna, esto al institucionalizar otras formas de relacionalidad o "convivialidad" para transformar la sobrevida miserable que sufre gran parte de la humanidad (1).

En este sentido, el respeto de la dignidad humana, la igualdad de derechos y la justicia son las únicas garantías de la paz en el mundo, campo en el cual confluye la bioética con la garantía de los derechos humanos, en la satisfacción de las necesidades y de las libertades básicas (2). Por tanto, es necesario desde la bioética abordar las desigualdades socioeconómicas y en salud, aspectos sobre los cuales Daniel Callahan, hace más de cuatro siglos, presentó sus reflexiones demostrando la incidencia de estas desigualdades en la estructura, el financiamiento y la organización de los servicios de salud. Propuesta teórica que sirvió de fundamento para el desarrollo de la denominada bioética de la salud de las poblaciones, la cual se concentra en muchos factores que son determinantes de la salud; es decir, que no sólo interesa el acceso a los servicios, sino también a los factores que explican el por qué algunas personas se enfermen más que otras, tales como -las condiciones de salud, los valores que guían las políticas públicas y los macrodeterminantes de la salud- (3).

Para dimensionar este interés investigativo a la luz de la bioética es necesario precisar lo que se entiende como condición de MME, constituyéndose en latinoamericana en un evento de interés en términos de Salud Pública, sirviendo además, como una forma de monitorear el nivel de desarrollo integral de los países. Sobre el particular, el Modelo de vigilancia colombiano define la MME como "una grave complicación ocurrida durante el embarazo, parto y puerperio, que pone en riesgo la vida de la mujer y que requiere de una atención inmediata con el fin de evitar la muerte" (4). En este sentido, la Organización Mundial de la Salud (OMS) conceptualiza la MME como un estado en el cual una mujer casi muere, pero sobrevivió a una complicación ocurrida durante el embarazo, el parto o dentro de los 42 días de la terminación del embarazo. Por su parte, la Federación Latinoamericana de Sociedades de Obstetricia y Ginecología (FLASOG) añade otras características como el ser ésta una complicación grave que ocurre durante el embarazo, parto y puerperio, que pone en riesgo la vida de la mujer o requiere una atención inmediata con el fin de evitar la muerte. Ampliando un poco más estos conceptos, se añaden los criterios de inclusión de los casos de MME que abarcan condiciones clínicas como: pre-eclampsia severa, eclampsia, sepsis o infección sistémica severa, hemorragia obstétrica severa, ruptura uterina, disfunción orgánica, necesidad de transfusión, necesidad de atención en Unidad de Cuidados Intensivos -UCI-necesidad de procedimiento quirúrgico de emergencia, entre otras (5).

Conviene precisar que la interpretación de las narrativas sobre las experiencias vividas por las mujeres sobrevivientes a la MME teniendo en cuenta las categorías de análisis bioético que abarcan las etapas preconcepcional, prenatal, el nacimiento y el posparto, evidencia que en su conjunto constituyen el habla de la salud de la mujer, denominada salud materna (6). En la etapa preconcepcional se consideran los programas o servicios de atención de salud diseñados para ayudar a las parejas en la planificación del tamaño de la familia, mediante la utilización de varios métodos de anticoncepción para 
controlar el número de hijos, así como el momento ideal de los nacimientos (6). La etapa prenatal incluye la atención que se le proporciona a la mujer embarazada con el objetivo de prevenir complicaciones y disminuir la incidencia de mortalidad materna y perinatal (6). En la etapa del nacimiento se describe el desarrollo de un parto obstétrico -la expulsión del feto y la placenta bajo el cuidado del obstetra o de un asistente sanitario. Este proceso puede incluir intervenciones físicas, psicológicas, médicas o quirúrgicas- o bien por cesárea -que consiste en la extracción del feto mediante histerectomía abdominal- (6). Finalmente, la etapa del puerperio comprende el periodo de posparto; es decir, el tiempo que trascurre hasta los 42 días después del parto (6). Se debe aclarar, que aunque estas etapas son definidas teniendo en cuenta aspectos clínicos, es preciso mencionar que los desenlaces que se dan en cada paciente están estrechamente relacionados con sus condiciones de vida y con la posibilidad de acceso a los servicios de salud. De ahí que, este tipo de características se tuvieran en cuenta para configurar la información de interés, partiendo de las narrativas de las mujeres.

En general, la mujer que vivió una MME en el proceso de atención necesitó transfusiones sanguíneas, necesidad de atención en UCI o de procedimiento quirúrgico de emergencia, entre otros procedimientos, considerados de alta complejidad. En este contexto el poder entender a la paciente sobre sus experiencias requiere hablar con ellas; es por eso que Cardona reflexiona sobre la metáfora de la enfermedad, la cual integra cómo asumir y comunicar la cruda vivencia de la enfermedad y el dolor, sintiendo una necesidad de comunicar a otros lo vivido, lo cual es íntimo e individual. Este enfoque permite comprender el mundo de cada persona; es decir, que el lenguaje traslaticio de la metáfora permite redefinir las posibilidades y victorias frente a la experiencia del dolor y la enfermedad (7).

También, el vivenciar estos procesos salud - enfermedad implica en las mujeres una serie de consideraciones en relación a cómo ejercen su autonomía. En este sentido Peluchon habla de la autonomía quebrada, la cual si es mirada sólo desde el abordaje de la autodeterminación, es inutilizable en medicina, pues la enfermedad causa numerosos efectos sobre las personas, ya que además de comprometer esta autodeterminación, no permiten pensar en la autonomía de una persona en situación de dependencia y de vulnerabilidad (8). Las mujeres sobrevivientes a la MME frecuentemente están en situaciones tan críticas que no pueden tomar decisiones sobre su salud, momento en el cual se acude a familiares para este abordaje. Sin embargo, muchas veces aunque pueden estar aparentemente conscientes, el dolor, el cansancio, el estrés, la falta de entendimiento sobre las implicaciones de su estado de salud y muchos otros aspectos, influyen en que las decisiones que tomen estén en el marco de una autonomía quebrada; es decir, se encuentran en contextos donde esa autodeterminación no se puede ejercer en su totalidad a causa de la enfermedad.

En este contexto, las categorías de análisis bioético desempeñan un rol central, pues por su medio se puede hacer una interpretación al tener como cimiento los aspectos relacionados con lo dialógico y la orientación de la bioética. Es desde esta perspectiva, que varias categorías de análisis bioético se destacan por sus potenciales aportes en la interpretación de la experiencia vivida al ser mujer en condición de MME, tales como: la equidad, la autonomía, la justicia, la concepción del bien y el mal, el acceso a los servicios de salud, las condiciones de vida desde la pluralidad cultural, la comunicación, el lenguaje y la deliberación bioética; siendo esta última una herramienta que permite circunscribir estas categorías a problemáticas concretas.

\section{Desarrollo}

\section{Necesidad de abordar la MME desde la bioética}

La Organización Mundial de la Salud (OMS) estima que cada día mueren cerca de 800 mujeres por causas prevenibles relacionadas con el embarazo y el parto, 99\% de estas ocurren en países de ingresos medios y bajos (9). En Colombia la razón de mortalidad materna ha disminuido de forma significativa en las últimas décadas pasando de 100/100.000 Nacidos Vivos (NV) en 1998 a menos de 60,4/100.000 NV después del año 2010; en relación con la MME para el año 2016 fueron reportados 31 casos por cada $1000 \mathrm{NV}$ (10). Estos desenlaces desfavorables en salud materna se presentan de forma persistente en las regiones del país donde hay mayor población con necesidades básicas insatisfechas y con más bajos niveles de educación (11).

Conforme a la caracterización anterior, es evidente que en Colombia las mujeres que sufren con mayor frecuencia de estos desenlaces en salud están inmersas en entornos socioeconómicos desfavorables, lo cual permite claramente hablar de tres categorías de análisis bioético: la equidad, la justicia y la autonomía. Al respecto, considerando la MME desde la perspectiva de la macrobiótica o bioética global, se pueden mencionar los aportes que Iván Illich hizo al reconocimiento de estas categorías de análisis bioético -equidad, justicia y autonomía-. Según este autor en un contexto de crisis 
Cienciä@Cuidado

Scientific Journal of Nursing
Categorías bioéticas en la interpretación de ser

mujer con morbilidad materna extrema de la sociedad industrial sin límites y desproporciona$\mathrm{da}$, propone estos criterios para que las tecnologías e instituciones humanas dominantes o "las herramientas dominantes" tengan límites y proporciones; es decir, deben ser "convivenciales", que sean parte de un nuevo talante moral que equilibre lo individual -de corto plazo- y las necesidades a largo plazo de la especie humana y que garanticen una supervivencia decente (12).

De acuerdo con el nivel macro, entendido como la forma en que los gobiernos organizan sus recursos y disponen sus políticas públicas, así como el nivel intermedio que hace referencia a la forma como las instituciones prestadoras de servicios de salud priorizan y brindan sus servicios, se evidencia que en Colombia ambos niveles están influenciados de forma directa por esas herramientas dominantes, lo que se traduce en las desigualdades en el acceso a los servicios de salud materna desde la pre-concepción, pasando por la gestación, hasta el parto.

En función de lo planteado anteriormente, desde este enfoque de la bioética existe una preocupación por analizar la distribución de la salud universal, a partir de una perspectiva de salud amplia, no individual sino poblacional; parámetros que permiten tener en cuenta los determinantes sociales desfavorables que influyen en aspectos como la salud y el acceso a los servicios de atención en salud; factores que inciden en que unas personas se enfermen más que otras, orientando por tanto, el interés en los menos favorecidos (13).

Por tanto, al hablar desde la bioética de esas desigualdades existentes en el acceso a la salud materna en Colombia, es indudable que muestran la ausencia de equidad en salud, escenario en el que es importante tener una visión no tanto teórica, sino proactiva. En este sentido, Lolas F explica que en un contexto de realidades sociales que son cambiantes no hay que perder de vista aspectos como la distribución del poder, pues la reflexión y la acción bioética no solamente deben seguir a los avances científicos, sino anticiparlos y examinar los escenarios que los posibilitarán, ya que solo de esta forma la reflexión bioética será un componente sustantivo de las etapas iniciales en que los determinantes macrosociales de la salud y sus perturbaciones son establecidos (14).

Al respecto, los posibles impactos y riesgos de la globalización sobre estos determinantes macrosociales de la salud, se relacionan con lo que exponen Chen y Berlinguer para quienes con la aparición de nuevas amenazas sanitarias y ambientales, la disparidad entre países -unos muy pobres y otros muy ricos-, implica que, para lograr políticas sociales tendientes a la equidad mundial en salud, son necesarias instituciones fuertes en las esferas comunitaria, nacional y mundial (15).

\section{La MME desde la visión del bien, del mal y del poder}

En torno a la concepción del bien y el mal como categoría de análisis bioético, es importante tener en cuenta algunos elementos que aporta Badiou a la interpretación de la experiencia vivida por las mujeres sobrevivientes a la MME. El primer elemento se relaciona con la concepción que da el autor sobre la ética, para quien la ética debe ser más activa, más vivida y no solo un discurso (16). Es decir, que esta ética viva necesita trascender las dinámicas en el día a día en los procesos de atención en salud, tales como el respeto a la autonomía de la mujer frente a sus decisiones sobre su salud.

Para ejemplificar esta afirmación, se considera el caso de una niña que crece en condiciones de vulnerabilidad socioeconómica, lo que supone reflexionar en torno a la libertad que tiene para ejercer su autonomía en decidir sobre su proyecto de vida y así planificar si tener o no hijos y cuándo hacerlo. En consecuencia, debe tener acceso oportuno a servicios en salud sobre la pre-concepción y durante la gestación, lo cual aún es todo un desafío para el sistema de salud colombiano. De acuerdo con Badiou cada sujeto es singular y construye sus realidades y los simulacros de la verdad en que vive de acuerdo con sus contextos políticos, científicos, artísticos y amorosos, pues "no hay un solo sujeto, sino tantos como verdades hay" (16). Por tanto, la pregunta que surge a la luz de estas reflexiones es cómo se abordan desde los servicios de salud materna esas singularidades, o más bien, de qué forma se prestan esos servicios estandarizados, que muchas veces no son sensibles a las necesidades sentidas por estas mujeres.

Es necesario recalcar que las mujeres que son sobrevivientes a la MME viven situaciones sobre la verdad o realidades en donde no pueden ejercer con libertad su autonomía, a consecuencia de determinantes sociales sobre salud desfavorables y que están presentes en su contexto. En este punto, es importante hablar de la percepción que sobre el bien y el mal tiene Badiou, quien precisa que desde el nivel macro muchas veces con base en una justificación ética de hacer el bien a una comunidad con el ideal de ser en conjunto, se hacen acciones individuales y colectivas que pueden lesionar a otros (16).

En esta visión macro, se puede reflexionar en torno a la raíz de las dinámicas nacionales que vulneran la atención a la salud materna, dado que este accionar sig- 
nifica que en Colombia se prioriza este beneficio en favor de las personas más favorecidas del país. De igual forma, en el nivel micro, a partir de la relación personal salud - paciente, hay que auspiciar una ética más viva y más consciente, puesto que se observa deshumanización en los servicios de atención materna. Esta falta de equidad se advierte en las rutinas y prácticas en donde a la mujer en proceso de parto no se le atiende como un ser humano, sino como un número más, despersonalizándola y convirtiéndola en un objeto. En función de lo expuesto, no solo se debe cambiar la forma en que se percibe y vive la ética desde los servicios de salud; sino que también hay que revisar en qué punto se pierde esa consciencia crítica para ejercer los principios de beneficencia, sobre todo en lo relacionado con las profesiones de las ciencias de la salud.

En el marco de esta problemática, al hablar de la concepción sobre el bien y el mal y su aplicación en el contexto donde se desarrolla la MME se pueden tomar como referentes los aportes de Arendt $\mathrm{H}$ quien reflexiona respecto a la banalidad del mal, en su análisis sobre el caso Nazi. Para Arendt, cuando se está en un entorno en el que los criterios morales en la vida pública y privada colapsan, las personas son proclives a que se dé un colapso también de su juicio personal, de tal suerte que la moral pasa a ser solo un conjunto de costumbres y hábitos que son aprendidos y replicados para obtener la aprobación de la sociedad en la que se está, de tal suerte que se puede llegar al punto de cometer hasta delitos graves, sin saber o intuir que se están realizando actos de maldad (17).

Lo anterior, se torna peligroso al hablar de la relación del personal de salud con el paciente en los servicios materno-perinatales, en donde la deshumanización de la atención en salud se da, porque existen contextos laborales y dinámicas por las cuales muchos de los profesionales de la salud en un esfuerzo de supervivencia, los asumen como actos rutinarios y con el objeto de cumplir estándares establecidos, que les permitan tener aprobación como trabajadores eficientes; de ahí que terminen instrumentalizando y despersonalizando a quienes son sus sujetos de cuidado. En esta situación a las mujeres se les llamará "mamá" o "mamita" o por el número de su cama, dando origen a dinámicas por las cuales las pacientes que están viviendo su proceso de parto, nacimiento, su recién nacido y su familia, no son tratadas como seres humanos

En este recorrido por las categorías de análisis bioético en la interpretación de la condición de la MME, el uso del poder se constituye en un determinante de las políticas públicas en salud materna en los diferentes estados, garantizando en mayor o menor proporción la equidad, la autonomía y la justicia. Al respecto, Foucault considera que en las sociedades existen mecanismos y dispositivos de poder que se desarrollan por medio de procedimientos que quieren dominar los acontecimientos, siendo los cuerpos de los seres humanos los objetivos de ese poder, sobre los cuales se ejerce coerción, la cual muchas veces es tan sutil que no son evidentes los límites entre el controlado y el controlador (18).

Desde esta perspectiva, los servicios de atención a la maternidad se convierten en dispositivos que ejercen ese poder sobre las mujeres, siendo notorias las relaciones de poder que históricamente se dan entre pacientes y personal de salud, contexto en donde la posición de la mujer en proceso de parto no es ni natural ni beneficiosa, sino la más cómoda para el personal de salud.

Categorías de análisis aportadas desde la bioética de las poblaciones y la bioética de intervención en la interpretación de la MME

Desde una perspectiva amplia de la bioética, la interpretación de la MME puede apropiarse de elementos teóricos a partir de la bioética de la salud de las poblaciones, que de acuerdo con Daniel Wikler, se enfoca en aspectos como los determinantes de la salud, el acceso a los servicios de atención en salud y los factores que generan que unas personas se enfermen más que otras (19). Wikler reflexiona y expresa que la bioética necesita nuevos recursos filosóficos, que vayan más allá de la moralidad individual o de los códigos éticos, más bien se necesitan elementos sociales y políticos, particularmente desde las teorías de la justicia distributiva, pues los problemas en la salud de las poblaciones necesariamente ocupan aspectos fundamentales como la economía, la historia y la política, los cuales determinan directamente el cuidado de la salud y generan desigualdades que determinan las condiciones de vida de las poblaciones y las posibilidades de acceso a los servicios de salud (19).

Este autor propone que la bioética se ha desarrollado en cuatro momentos, empezando por los códigos de conducta profesional, seguidos del surgimiento de la bioética en la relación médico-paciente y posteriormente está el enfoque de la estructura, el financiamiento y la organización de los servicios de salud. Como última fase se centra en una bioética preocupada por la salud de la población (13). Con respecto a este último aspecto expone que la salud de las poblaciones se relaciona y se ve impactada por las tecnologías en salud, por las condiciones de acceso a los servicios de atención en salud, por los factores que generan que unas personas 


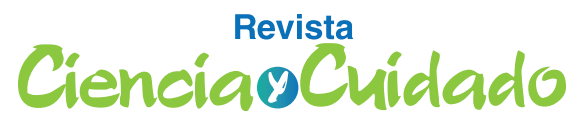

Scientific Journal of Nursing
Categorías bioéticas en la interpretación de ser mujer con morbilidad materna extrema se enfermen más que otras, por las condiciones de los menos favorecidos y como estos necesitan ser priorizados sobre el resto de la población (13).

Teniendo en cuenta los aspectos mencionados anteriormente que confieren características diferenciadoras a las realidades y contextos que se viven en la región; sin embargo, existen posiciones consolidadas desde la bioética Latinoamericana que proponen otras categorías de análisis a ser consideradas además de los cuatro principios tradicionales -autonomía, beneficencia, no maleficencia y justicia-. En este aspecto uno de los pensadores destacados en esta materia es Volnei Garrafa, quien propone otras herramientas teóricas y metodológicas adicionales capaces de enfrentar los macro-problemas, ya sean históricamente persistentes o emergentes en los diferentes ámbitos, teniendo como base el respeto al pluralismo moral, con un enfoque esencialmente transdisciplinar. Según este autor se debe tener en cuenta la visión de las cuestiones a partir de los paradigmas de la complejidad y la totalidad concreta, referidos por el análisis bioético como la comunicación, el lenguaje, la argumentación, el diálogo, la coherencia, el consenso y la racionalidad (20). Sobre estas bases Volney Garrafa propone la denominada bioética de la intervención la cual se centra en la justicia y la salud pública, y se relaciona con la filosofía del Buen Vivir es decir, que-todas las personas tienen derecho a una vida decente, que les asegure salud, alimentación, agua limpia, oxígeno puro, vivienda adecuada, saneamiento ambiental, educación, trabajo, descanso, ocio, cultura física, vestuario, pensión, etc.--Según esta teoría no se consideran solamente los bienes materiales, sino que da más relevancia a aspectos como el conocimiento, el reconocimiento social, la pluralidad cultural, los códigos éticos y espirituales de conducta, la relación con la naturaleza, los valores humanos y la visión de futuro (21).

Para la interpretación de los significados de la experiencia vivida de la MME se toman como referentes de análisis de la bioética de intervención planteada por Garrafa y colaboradores conceptualizada como la comunicación / el lenguaje y la pluralidad cultural -el relativismo ético o la no universalidad de los principios-. Para estos autores el término "comunicación" designa el carácter específico de las relaciones humanas que comprenden los intercambios con participación recíproca y/o comprensión; además, indica un conjunto de modos específicos que la coexistencia humana puede asumir. Por su parte el "lenguaje" consiste en el vocabulario específico usado para expresar ideas, sentimientos, comportamientos. Estos dos términos son esenciales al aplicarlos al ámbito de la salud materna, por ejemplo en casos puntuales, como es la aplicación del proceso de consentimiento informado en relación a procedimientos o tratamientos clínicos (21).

En este mismo sentido, en lo que se refiere al análisis de la pluralidad cultural basada en el relativismo ético, se sabe que se centra en la no universalidad de los principios bioéticos. Al respecto autores de diferentes culturas proporcionan visiones también diversas frente a un mismo hecho o situación; lo que significa que al imponer una visión moral de una cultura o de una nación políticamente más fuerte frente a otra más débil, da origen a lo que conoce como imperialismo ético o imperialismo moral. Desde este punto de vista, los cuatro principios de Georgetown -autonomía, beneficencia, no maleficencia y justicia- no pueden considerarse como universales. Es decir, que los análisis de los conflictos morales en diferentes culturas, necesitan estar contextualizados, pues las moralidades son relativas a cada lugar, a cada contexto biológico y socio-político-cultural (21).

Es así como para la presente investigación son pertinentes los elementos que ofrecen tanto la bioética de las poblaciones como la bioética de intervención, ya que es necesario realizar un análisis contextualizado que tenga como centro la justicia. Igualmente se deben integrar todas las características propias de la salud poblacional y la salud pública, incluyendo la forma como se da la comunicación en los servicios de salud donde se atiende a las mujeres; además hay que identificar cómo se dan las interacciones entre la pluralidad en las condiciones de vida y culturales de las pacientes -que pueden llamarse condiciones del buen vivir-. También, se debe tener muy en cuenta lo socioeconómico que abarca toda su integridad, permitiendo que en el acceso a los servicios de salud materna, se eviten desenlaces desfavorables en el tratamiento de la salud materno - perinatal. Por eso es importante prevenir ésta problemática, para que no se constituya en una macro problemática históricamente persistente en el contexto Colombiano.

Conviene subrayar, que al interpretar las experiencias de las mujeres que han experimentado la condición de MME es necesario examinar estas vivencias individuales desde la ética y la bioética a la luz de las características macro de la población a la que cada mujer pertenece. Se debe agregar que el análisis debe incluir sus condiciones de vida y de acceso a los servicios de salud preconcepcional y materno-perinatal, pues cuando estas condiciones son precarias en términos socioeconómicos, educativos y de ubicación geográfica les ubican en una franja poblacional con mayor vulnerabilidad y riesgo de presentarse desenlaces desfavorables. 
En este punto, es necesario mencionar a Rebecca Cook y sus aportes respecto a sus reflexiones éticas sobre la salud materna, desde el enfoque de la injusticia. Según esta autora los desenlaces desfavorables en salud materna son la expresión de las inequidades de género y de las injusticias que las mujeres vivencian al no tener el poder de autodeterminarse; es decir, que no pueden ejercer su autonomía, pues la vulnerabilidad social, económica y religiosa afectan su libertad de decidir, iniciar o continuar su gestación. Además, tiene que ver con el disfrute de un acceso apropiado a los servicios de salud prenatal, durante el parto y en el posparto. Lo que significa que los servicios de salud no resuelven los problemas de injusticia en cuanto a una maternidad segura, puesto que se relacionan directamente con factores condicionantes que hacen que la mujer no pueda regular su salud reproductiva y su vida sexual. Todo lo anterior es el resultado de una discriminación sistemática contra la mujer en la que los aspectos políticos, los económicos, los religiosos y los judiciales están implicados (22).

Esta autora también afirma que casi el 99\% de las muertes maternas ocurren en los países en desarrollo y la diferencia entre el riesgo de muerte de una mujer de acuerdo a la región donde viva es más que dramática. Cook ejemplifica al comparar el riesgo de muerte de las mujeres durante la maternidad en Africa (1 de cada 20) comparada con las mujeres en América del Norte (1 de cada 4000). Este paralelismo es éticamente y legalmente indefendible ante las obligaciones de los países con la salud de la mujer; son diferencias que también se presentan al comparar en un mismo país poblaciones con mayor y menor grado de vulnerabilidad. En este contexto Cook reflexiona desde la perspectiva de la ética sobre aspectos como la justicia en términos de la no discriminación, los derechos de igualdad en términos de la asignación de recursos sanitarios y la garantía de los derechos humanos como lo es el derecho a la supervivencia -a la vida- (23).

Desde otro punto de vista Cook establece que los derechos humanos deben estar garantizados mediante una maternidad segura, siempre en función de las siguientes categorías: 1. relacionados con la vida, la libertad y la seguridad de la mujer; 2. relacionados con la fundación de las familias y la vida familiar; 3. relacionados con el acceso a la salud y a los beneficios de los progresos científicos, incluida la información y la educación en salud; y 4. los relacionados con igualdad y la no discriminación por sexo, estatus marital, raza y clase social (24).

Además, agrega Cook que los profesionales de la salud tanto a nivel colectivo como individual, deben tener un rol activo frente a las injusticias sociales que afectan a las mujeres en sus vidas por causas prevenibles; esto se logrará con argumentos éticos y la aplicación de medios legales que pueden ser realizados por las organizaciones profesionales que tienen el deber macro ético para con los pacientes y así promover la supervivencia y la salud de los pacientes (23).

Se debe precisar que los aportes de Cook y Dickens en la interpretación del significado de la experiencia que viven las mujeres que desarrollaron $\mathrm{MME}$ se centran en la articulación de categorías de análisis ético como son: la autonomía, la justicia y la equidad. Igualmente lo hacen a partir del enfoque de los derechos humanos, integrando reflexiones sobre aspectos estructurales de las dinámicas de la atención materno - perinatal, que incluyen aspectos como la asignación de recursos y el rol de los estados en la garantía de una atención materno - perinatal segura, por lo cual estos aportes se constituyen en bases importantes para el desarrollo de la presente investigación.

De forma más específica Urrea-Mora F es una investigadora de referencia que a nivel nacional aporta argumentos en torno a las reflexiones bioéticas durante la gestación, teniendo como categorías de análisis: la maternidad, el cuerpo y la autonomía en un diálogo bioética-género. Del mismo modo expone sus aportes en términos de bioética, género y bioética-género, respetando la autonomía de las mujeres gestantes durante la atención en salud; actitudes que están mediadas por los significados que del cuerpo y la autonomía femenina tienen tanto las mujeres como los profesionales de la salud que las atienden (25).

Sobre el género esta autora afirma que la problemática radica en que el cuerpo de las mujeres ha tenido una connotación de inferioridad por ser diferente, razón por la cual se la ha excluido y discriminado a lo largo de la historia hasta hoy (26); además, argumenta que la autonomía, la justicia y el género se entretejen como elementos que favorecen a los sujetos autónomos, quienes a su vez reclaman justicia social (27). En este sentido, agrega que la atención clínica de los profesionales de salud se centra en el sistema reproductor de las gestantes y los riesgos del embarazo, convirtiendo al cuerpo en un objeto, instaurando relaciones paternalistas que vulneran la autonomía de las mujeres -la capacidad del ser humano para decidir y "construir" su contexto(28).

Para finalizar y una vez revisada la literatura sobre esta temática desde diferentes visiones científicas, permite reflexionar en torno a las categorías de análisis que son 


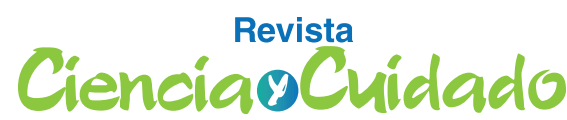

Scientific Journal of Nursing aplicables en la interpretación de los significados de la experiencia vivida al ser mujer en condición de Morbilidad Materna Extrema (MME). Al respecto, se percibe que desde la bioética de la salud de las poblaciones, las categorías justicia - equidad, autonomía y acceso a los servicios de salud, así como desde la bioética de intervención se deben dar condiciones de vida desde la pluralidad cultural, teniendo en cuenta la comunicación y el lenguaje.

\section{Conclusiones}

De forma general, se habla de la aplicación de las categorías de análisis bioético para interpretar los significados de las experiencias que vivieron las mujeres sobrevivientes a la MME, categorizadas previamente como: -justicia / equidad, autonomía y acceso a los servicios de salud; así como la visión desde la bioética relacionada con la intervención de las condiciones de vida desde la pluralidad cultural y las consideraciones en torno a la comunicación y el lenguaje- las cuales deben pensarse en conjunto desde una visión proactiva de la bioética, que permita analizar y generar acciones de impacto que influyan en los casos de las mujeres como seres individuales, en acciones de política pública tanto regionales como nacionales que mejoren su salud pre-concepcional y de gestación.

En este escenario tan desafiante, son muy importantes las propuestas que hace Cortina A sobre una bioética cívica global que aborde los problemas de la vida desde diferentes niveles tales como: la macrobioética -global-, la mesobioética -estados nacionales- y lo microbioético -bioética clínica- (29). En todo caso, estos abordajes tienen que ser pensados e implementados en los contextos particulares de cada mujer; de ahí, que deban ser individuales, puesto que en muchos casos la grave- dad e implicaciones de la MME se dan en ambientes de vulnerabilidad, ocasionando desenlaces desfavorables. Desde este punto de vista, se sabe que esta experiencia representa un cambio de rol para la mujer quien además de sufrir transformaciones físicas, también percibe una variedad de emociones (30) frente a este tipo de enfermedad.

En estos niveles, es necesaria la aplicación de una bioética dialógica y cordial, que permita deliberar en torno a asuntos relacionados con la salud materna contando siempre con la voz de todos los implicados (29). Por tanto las mujeres, las familias, las instituciones y las autoridades, entre otros- deben estar implicados en aspectos coyunturales relacionados con preguntas sobre ¿qué normas son equitativas?, ¿cómo estructurar instituciones equitativas?, ¿cómo abordar los problemas en salud materna? y ¿cómo mitigar el desarrollo de la MME?, siempre en función de parámetros que tienen que ver con la deshumanización de los servicios de salud y las problemáticas en cuanto a la aplicación del consentimiento informado, entre otros.

\section{Agradecimientos}

A los docentes que orientan las actividades académicas del Doctorado en Bioética de la Universidad Militar Nueva Granada. A la Universidad de Boyacá por permitirme el desarrollo de estas actividades académicas.

\section{Conflictos de interés}

La autora declara la no existencia de conflictos de interés ya sea por motivos de financiación del proyecto del cual este artículo es su resultado ; así como por motivos intelectuales, académicos, morales e investigativos.

\section{Referencias Bibliográficas}

1. Osorio-García SN. El desafío bioético de la era planetaria: La Convivialidad. Revista Latinoamericana de Bioética [Internet]. 2009 [consultado 01 de octubre de 2020]; 9 (2): 42-61. Disponible en: https://www.redalyc.org/ pdf/1270/127020306004.pdf

2. Organización de las Naciones Unidas para la Educación, la Ciencia y la Cultura - UNESCO. Justicia y Derechos Humanos: Justicia, igualdad y equidad. En: Tealdi, Juan-Carlos. Diccionario Latinoamericano de bioética. 1ra edición [Internet]. Colombia: Editorial Universidad Nacional de Colombia; 2008 [consultado 01 de octubre de 2020]: 372-383. Disponible en: http://redlaceb.com/diccionario-latinoamericano-bioetica/

3. Ferrer LM. Equidad y justicia en salud implicaciones para la bioética. Acta bioeth [Internet]. 2003 [consultado 01 de octubre de 2020]; 9 (1): 113-126. http://dx.doi.org/10.4067/S1726-569X2003000100011. Disponible en: 
Scientific Journal of Nursing

https://scielo.conicyt.cl/scielo.php?script=sci abstract\&pid=S1726-569X2003000100011\&lng=es\&nrm=iso

4. Ministerio de Salud y Protección Social - Fondo de Población de las Naciones Unidas -UNFPA-. Introducción. En: Modelo de vigilancia de la morbilidad materna extrema. 1ra edición. Colombia: Ministerio de Salud y Protección Social [Internet]; 2014 [consultado 01 de octubre de 2020]; p 11. Disponible en: https://www.minsalud.gov.co/sites/rid/Lists/BibliotecaDigital/RIDE/VS/PP/SM-Evaluacion-Modelo-vigilancia-morbilidad-materna-extrema.pdf

5. Instituto Nacional de Salud y Ministerio de Salud. Vigilancia y análisis del riesgo en salud pública - Protocolo de vigilancia en salud pública Morbilidad Materna Extrema. PRO-R02.0000-59 [Internet] V02 22-12-2017 [consultado 01 de octubre de 2020]; p 23. Disponible en: https:/www.ins.gov.co/buscador-eventos/Lineamientos/ Pro_Morbilidad $\% 20$ materna $\% 20$ extrema.pdf

6. Biblioteca Virtual en Salud -BVS-. Descriptores en Ciencias de la Salud -DeCS- [Internet]. 2020 [consultado 01 de octubre de 2020]. Disponible en: http://decs2020.bvsalud.org/E/homepagee.htm

7. Cardona-Suárez LF. Enfermedad y metáfora. Pensamiento. Revista De Investigación E Información Filosófica. 2020; 76(288), 89-111. https://doi.org/10.14422/pen.v76.i288.y2020.005

8. Pelluchon C. El ejercicio de la medicina: valores de los pacientes, normas de los profesionales, conflictos y deliberación pública. Revista Colombiana de Bioética [Internet]. 2011 [consultado 01 de octubre de 2020]; 6 (2): 139-159. Disponible en: https:/www.redalyc.org/pdf/1892/189222558010.pdf

9. Organización Mundial de la Salud (OMS). Mortalidad materna [Internet], 2020 [consultado 01 de octubre de 2020]. Disponible en: https://www.who.int/es/news-room/fact-sheets/detail/maternal-mortality

10. Narváez-Díaz NS. Informe del evento Morbilidad Materna Extrema, Colombia [Internet] 2016. 2017 [consultado 01 de octubre de 2020]. Disponible en: https://www.ins.gov.co/buscador-eventos/Informesdeevento/Morbilidad\%20materna\%20extrema\%202016.pdf

11. Jurado-Ocampo, N. Factores de riesgo para Morbilidad Materna Extrema en Gestantes [Tesis Especialización en Ginecología y Obstetricia]. Bogotá: Universidad del Rosario; 2014.

12. Osorio-García SN. Ética para la bioética y bioética para la ética. El aporte de Iván Illich para el surgimiento de la Bioética global, En: Osorio-García, SN, Sierra-Cuartas, CE, Macraigne, S. La Bioética a la luz de las epistemologías de segundo orden I: El aporte crítico de Iván Illich y Hans Jonas. 1ra edición. Bogotá: UMNG- AF \& M Producción Gráfica S.A.S. 2014, p. 135-168.

13. Mendieta-Izquierdo G, Joya-Ramírez NS, Cuevas-Silva JM. Salud, justicia social y equidad: alternativas bioéticas para América Latina. Revista Redbioética / UNESCO [Internet] 2019 [consultado 01 de octubre de 2020]; 1 (19): 171 - 186. Disponible en: https://redbioetica.com.ar/revista-redbioetica-unesco-no-19/

14. Lolas F. El desafío bioético de la equidad: su relevancia en salud pública. Rev. Esp. Salud Publica. [Internet] 2001 [consultado 01 de octubre de 2020]; 75 (3): 187-192. Disponible en: http://scielo.isciii.es/scielo.php?script=sci arttext\&pid=S1135-57272001000300003\&lng=es.

15. Chen LC y Berlinguer G. Equidad en la salud en un mundo que marcha hacia la globalización. En: Desafío a la falta de equidad en la salud (Eds): Evans, T. Washington, D.C: OPS; 2002, p. 36-48.

16. Badiou A. La ética. Ensayo sobre la conciencia del mal [Internet]. 2020 [consultado 01 de octubre de 2020]. Disponible en: http://www.reflexionesmarginales.com/pdf/19/Documentos/3.pdf

17. Estrada-Saavedra M. La normalidad como excepción: la banalidad del mal, la conciencia y el juicio en la obra de Hannah Arendt. Revista mexicana de ciencias políticas y sociales. 2007; 49(201), 31-53. https://doi. 
Scientific Journal of Nursing

org/10.22201/fcpys.2448492xe.2007.201.42590

18. Cruz JI. El pensamiento de Michel Foucault como caja de herramientas. Discusiones Filosóficas [Internet]. 2006 [consultado 01 de octubre de 2020].; 7 (10): 183-198. Disponible en: http:/www.scielo.org.co/scielo. php?script=sci arttext\&pid=S0124-61272006000100011\&lng=en\&tlng=es.

19. Wikler D. Presidential Address: Bioethics and Social Responsibility. Bioethics. 1997; 11(3-4), 185-192. https:// doi.org/10.1111/1467-8519.00057

20. Garrafa V, Osório-Azambuja L. Epistemología de la bioética - enfoque latino-americano. Revista Colombiana de Bioética. 2009; 4 (1): 73-92

21. Rivas-Muñoz F, Garrafa VF, Ferreira S, Nascimento W. Bioética de intervención, interculturalidad y no-colonialidad. Saúde e Sociedade. 2015; 24(Suppl. 1), 141-151. https://doi.org/10.1590/s0104-12902015s01012

22. Cook RJ, Dickens BM. Human rights to safe motherhood. International journal of gynaecology and obstetrics: the official organ of the International Federation of Gynaecology and Obstetrics. 2002; 76(2), 225-231. https:// doi.org/10.1016/s0020-7292(01)00568-9

23. Cook RJ, Dickens BM. Ethical and legal issues in reproductive health: Ethics, justice and women's health. International Journal of Gynecology \& Obstetrics. 1999; 64: 81-85

24. Cook RJ, Dickens BM. The Injustice of Unsafe Motherhood. Developing world bioethics. 2002; 2(1): 64-81

25. Urrea-Mora FC. Respeto a la autonomía de mujeres gestantes en salud: diálogo bioética-género. Revista Colombiana de Bioética. 2014; 9: 193-236

26. Urrea-Mora FC. El cuerpo de las mujeres gestantes: un diálogo entre la bioética y el género. Rev. colomb. Bioét. 2012; 7(1): 97-110

27. Urrea-Mora FC, Pinilla-Alfonso M. Bioética, género y justicia social: experiencia de un seminario. Revista Colombiana De Bioética. 2018; 13(3), 67-73. https://doi.org/10.18270/rcb.v13i3.2483

28. Urrea-Mora FC. Matices de la autonomía en mujeres gestantes durante la atención en salud: un diálogo bioética-género. Revista Colombiana De Bioética. 2015; 7(2), 89-106. https://doi.org/10.18270/rcb.v7i2.812

29. Cortina A. Bioética para el siglo XXI: construyendo esperanza. Revista Iberoamericana de Bioética. 2016; (1): 142-144. https://doi.org/10.14422/rib.i01.y2016.001

30. Escobar-Bermúdez A, Bejarano-Beltrán M. Vivencias de mujeres con preeclampsia en la Unidad de Cuidados Intensivos Obstétricos en Colombia. Enfermería Clínica. 2021; In Press. https://doi.org/10.1016/j.enfc$\underline{\text { li.2020.11.003. }}$ 